\title{
Application of particle swarm optimization with ANFIS model for double scroll chaotic system
}

\author{
W. A. Wali \\ Department of Computer Engineering, College of Engineering, Basrah University, Iraq
}

\begin{tabular}{l}
\hline \hline Article Info \\
\hline Article history: \\
Received Mar 21, 2020 \\
Revised Jun 22, 2020 \\
Accepted Jul 10, 2020 \\
\hline
\end{tabular}

\section{Keywords:}

ANFIS

Chaotic time series PSO

\begin{abstract}
The predictions for the original chaos patterns can be used to correct the distorted chaos pattern which has changed due to any changes whether from undesired disturbance or additional information which can hide under chaos pattern. This information can be recovered when the original chaos pattern is predicted. But unpredictability is most features of chaos, and time series prediction can be used based on the collection of past observations of a variable and analysis it to obtain the underlying relationships and then extrapolate future time series. The additional information often prunes away by several techniques. This paper shows how the chaotic time series prediction is difficult and distort even if neuro-fuzzy such as adaptive neural fuzzy inference system (ANFIS) is used under any disturbance. The paper combined particle swarm (PSO) and (ANFIS) to exam the prediction model and predict the original chaos patterns which comes from the double scroll circuit. Changes in the bias of the nonlinear resistor were used as a disturbance. The predicted chaotic data is compared with data from the chaotic circuit.
\end{abstract}

This is an open access article under the CC BY-SA license.

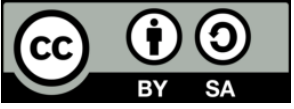

\section{Corresponding Author:}

Wasan Wali,

Department of Computer Engineering,

Basrah University,

Basrah, Iraq.

Email: wasan.wali@uobasrah.edu.iq

\section{INTRODUCTION}

Nonlinear prediction of chaotic time series is very challenging in the prediction area [1]. Neural networks and related neuro-fuzzy models such as (ANFIS) have been the subjects of interest due to their many practical applications in modeling complex nonlinear systems [2-4] and in chaotic time series predictions [5-10] but when the number of observations for training is limited or the data does not have a similar pattern they can neither reconstruct the dynamics nor learn the shape of attractor and the prediction become more difficult. In this paper, we used the asymmetrical double scroll attractor as an obstacle for the best technique which is the ANFIS model to predict the original pattern and propose the odd information which does not match with the original pattern. This oddness in information can be with purpose or as distortions. The paper shows how it is difficult and the distortions that happened on the perditions of chaotic attractor by using the ANFIS model under disturbance. Chaos in electrical circuits has drawn strong attention [10-15] since the circuit provides a simple vehicle for the experimental observation and computer simulation of chaotic phenomena. The double scroll circuit is used for nonlinear time series analysis methods for confirming the chaotic behavior Figure 1 shows the double scroll circuit, circuitry, and nonlinear resistor characteristic. Figure 2 shows symmetric double spiral attractor. 
Chaotic systems are sensitive dependency on initial conditions and densely distributed periodic points [16]. The data collected along inevitable noise makes the prediction of a chaotic system hard to achieve. This difficulty proposes the modelling chaotic time series. Artificial Intelligence techniques have gained significant importance in modelling because of their ability to learn from historical observations data and predict highly nonlinear systems. ANFIS is a type of system that incorporates neural and fuzzy logic. Neurons learn from experience and performed by optimization of the antecedent and conclusion parts parameters. This paper uses ANFIS to predict a time series generated by a double scroll circuit but using asymmetrical attractor as kind of the noise via generated asymmetrical data pattern and evaluates the ANFIS prediction in this case. Figure 3 shows the block diagram of ANFIS prediction.

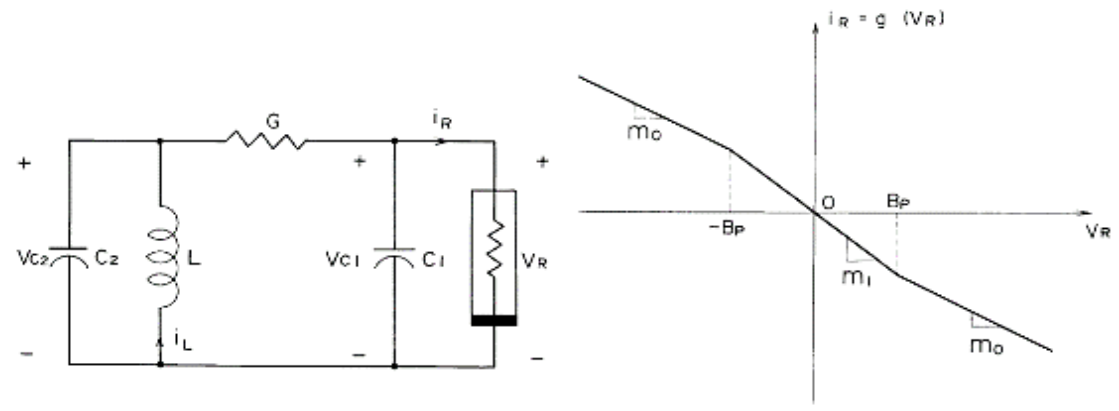

Figure 1. Double scroll circuit and nonlinear resistor characteristics

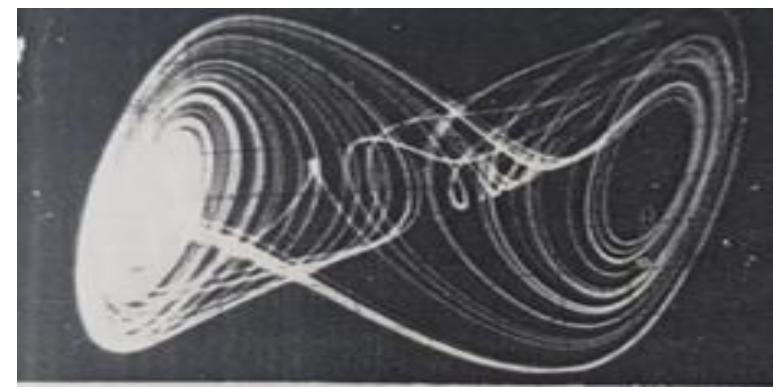

Figure 2. Symmetrical double strange attractor

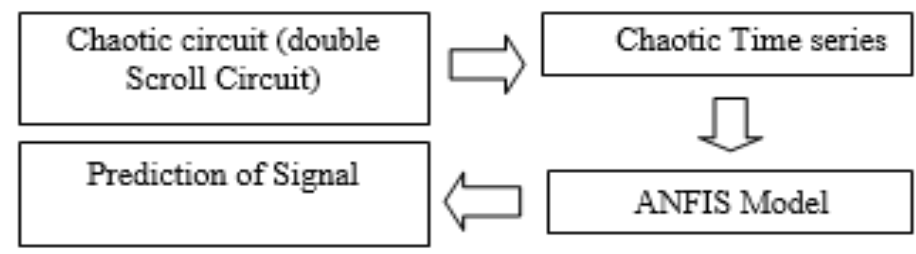

Figure 3. Block diagram of ANFIS prediction

\section{ASYMMETRICAL DOUBLE STRANGE ATTRACTOR}

Generally, the phenomenon of multiple attractors is mostly in symmetric dynamical systems [17-18]. These exhibit pairs of mutually symmetric attractors, as a parameter, are varied. The symmetry in double scroll strange attractor, which was observed from the electronic circuits, may not be held in special cases for various reasons, for example, switching characteristics of the diodes because of the differences in bias values or imperfect matching transistors or any other reasons that can make asymmetry in the strange attractor. Analysis of the nonlinear resistor circuit that is shown in Figure 4 indicates that the break voltage of the v-i characteristic is bias dependent. This can be related to the switching characteristics of the diodes. Thus it is expected that the bias sources $+\mathrm{Vcc}$ and $-\mathrm{Vcc}$, play a certain role as a bifurcation element. Figure 5 shows the effect of the bias on a double scroll attractor. 


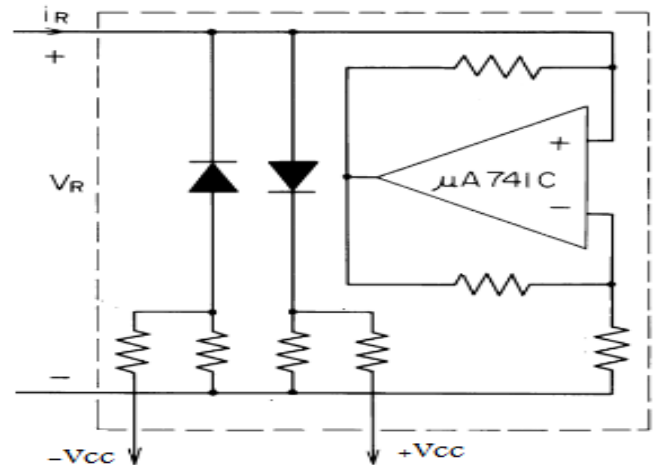

Figure 4. Nonlinear resistors
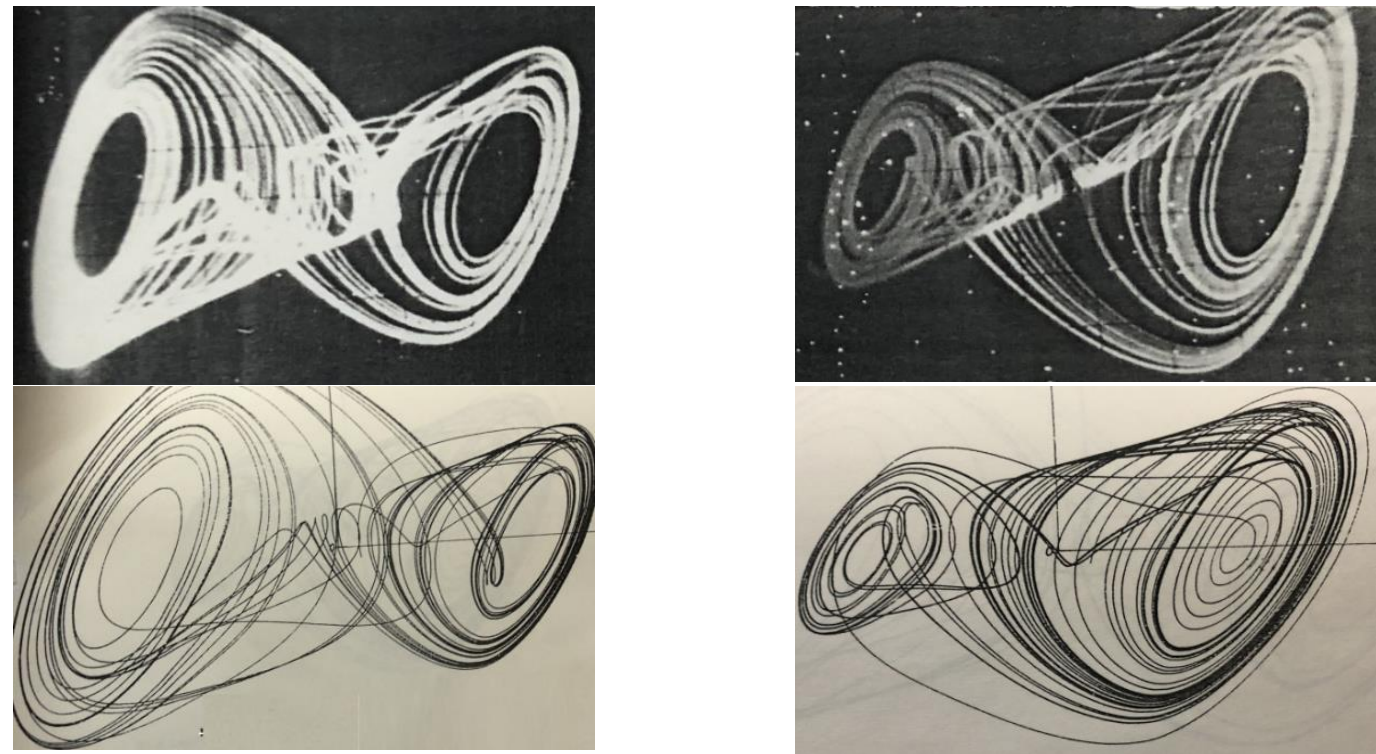

Figure 5. Asymmetrical double strange attractor

The prediction of a chaotic system from noisy observations is very hard. Noisy data could be contaminated from different types of sources. Noise can be propagated into the prediction model and make real problems in many of chaos real-life applications. Noise on chaotic time series prediction has been barely considered [19] to improve the performance prediction in the presence of noise. In this work, we focus on how to make a change in bias as disturbance data among the original data to examine the prediction of ANFIS based on chaotic noisy observations. Figure 6 shows the block diagram of the disturbance prediction system.

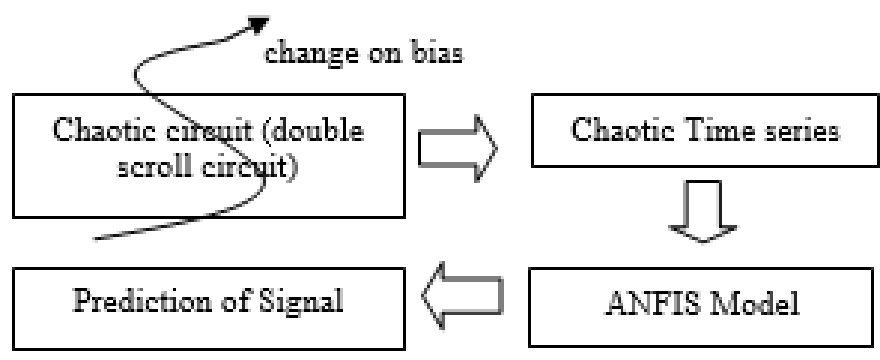

Figure 6. Disturbance on a chaotic system 


\section{COMBINED PSO WITH ANFIS TO REJECT THE BIAS DISTURBANCE}

The idea behind PSO is each particle keeps track of the coordinates in the space of the problem which is associated with the best solution [20]. Many studies have combined PSO with fuzzy and ANFIS in different applications [21-25]. In most of them the PSO tries to optimize the ANFIS parameters to give the best solutions. In our work, the PSO plays an important role in removing any undesired data and improving the prediction signal. PSO starts by taking the time-series data from the circuit under change as the initial first swarm generation. The closeness of each particle to the best solution depends on the objective function. The objective is the chaotic time-series data from the original double scroll circuit without any disturbance. Figure 7 and Figure 8 show the PSO-ANFIS model block diagram. Figure 9 shows the graphical representations. Considering the search space of 2-dimension and (n) particles as an initial population was taken from the circuit after the change of bias. There is a part of these particles that have a wrong position due to the change. Each particle has a specified position and velocity that is associated with its particular best performance in the swarm. Each particle tries to modify its position with its objective which is specified from the chaotic time-series data from the original double scroll circuit

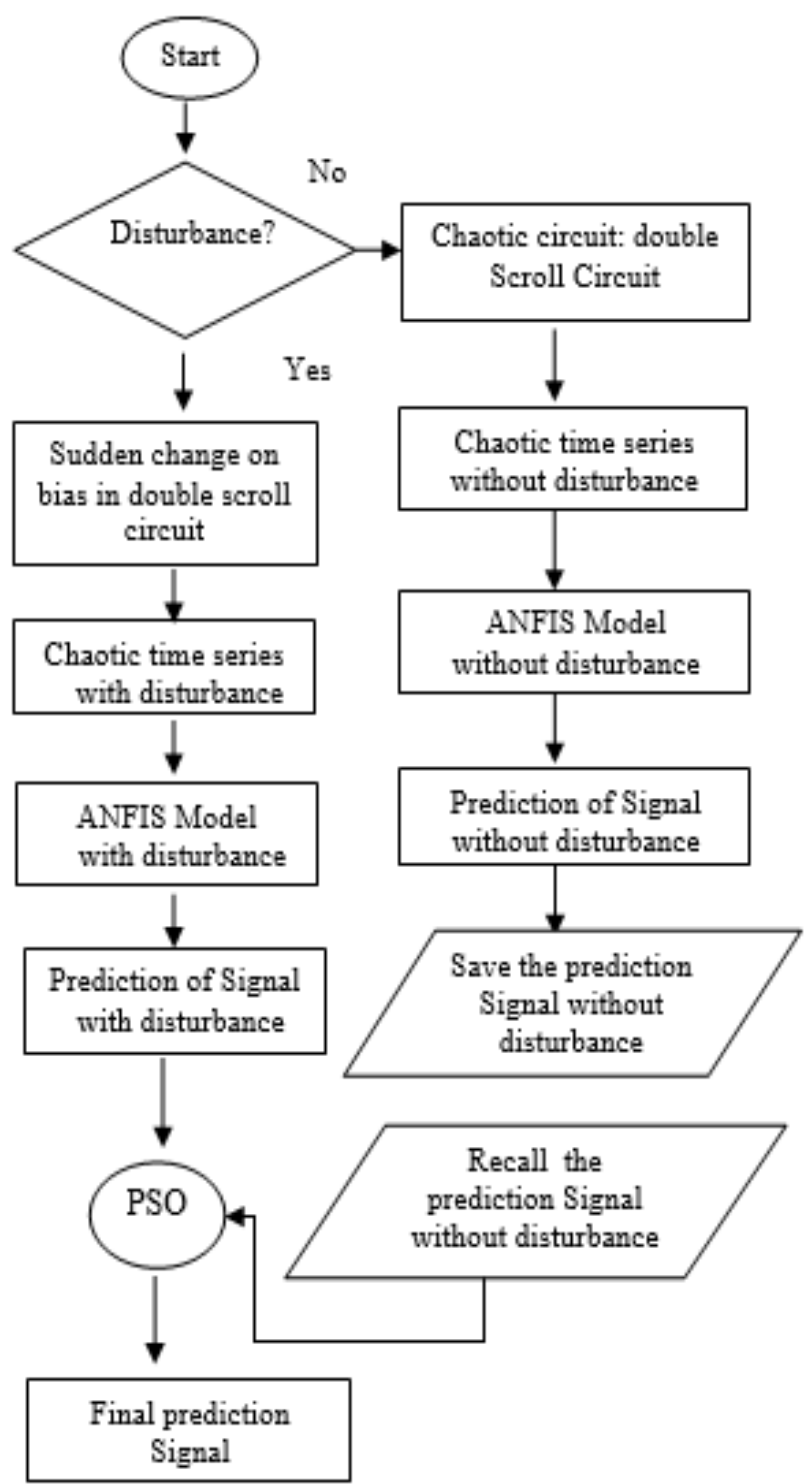

Figure 7. Disturbance on a chaotic system

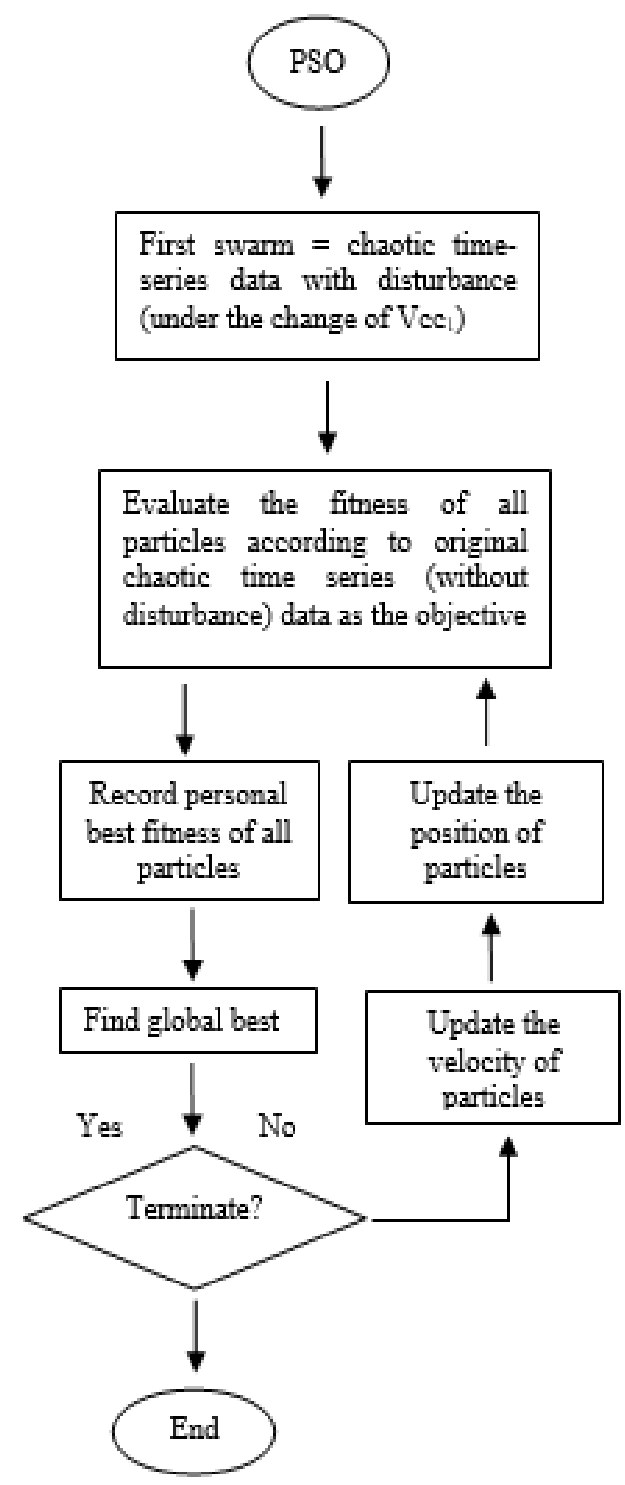

Figure 8. PSO-ANFIS prediction block diagram 

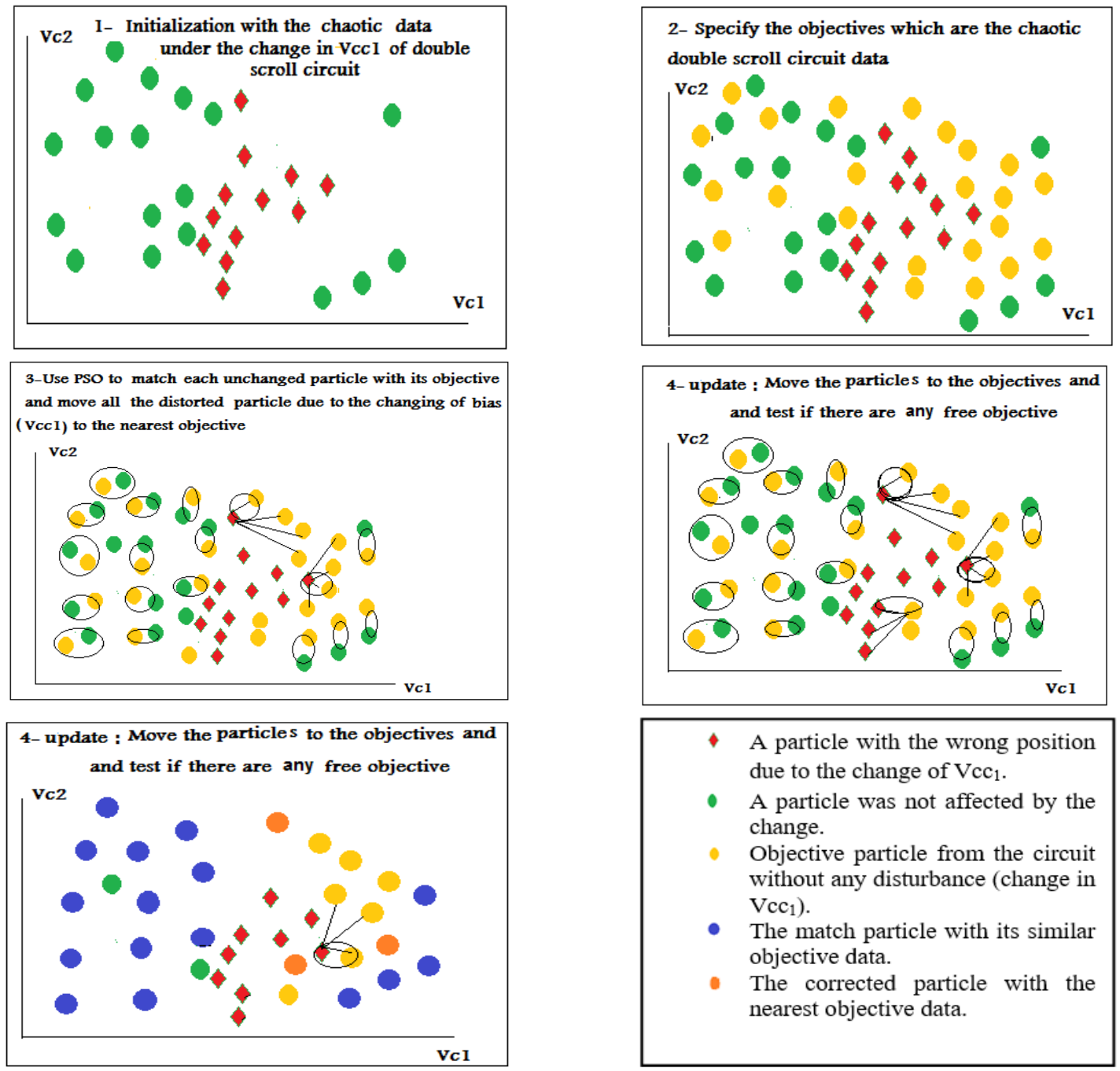

- A particle with the wrong position due to the change of $\mathrm{Vcc}_{1}$.

- A particle was not affected by the change.

- Objective particle from the circuit without any disturbance (change in $\mathrm{Vcc}_{1}$ ).

- The match particle with its similar objective data.

- The corrected particle with the nearest objective data.

Figure 9. PSO graphical representations

\section{RESULTS AND ANALYSIS}

The simulation was implemented using Matlab and the results arranged as the following:

- Generate the chaotic time-series data from the double scroll circuit $\left(\mathrm{Vc}_{1}\right.$ and $\left.\mathrm{Vc}_{2}\right)$ which show in Figure 10

- Make the change in $+\mathrm{Vcc} 1$ in nonlinear resistor which is shown in Figure 1

- Generate more chaotic data from the chaotic circuit after the change.

- Use ANFIS to predict chaotic data. Figure 11 shows the comparison between the simulations data from the circuit and predications of the ANFIS model. The figure shows the distortions in the attractor

- The distortions because of the additional changed data.

The simulation was implemented using Matlab and the results arranged as the following:

- Generate the chaotic time-series data from the double scroll circuit ( Vc1 and Vc2) which show in Figure 10.

- Make the change in $+\mathrm{Vcc} 1$ in nonlinear resistor which is shown in Figure 1

- Generate more chaotic data from the chaotic circuit after the change.

- Use ANFIS to predict chaotic data. Figure 11 shows the comparison between the simulations data from the circuit and predications of the ANFIS model. The figure shows the distortions in the attractor

- The distortions because of the additional changed data.

- Applied the PSO on the ANFIS model data. Figures 12, 13, and 14 show the ANFIS predictions model after PSO which applied according to the flowchart in Figure 7. 
- Figure 12 shows the particles update in their positions according to the individual nearest objectives taken from the circuit without disturbance.

- Figures 13 and 14 show PSO-ANFIS prediction on the attractor.

PSO-ANFIS can be used to predict and separate any kind of hidden pieces of information from any noisy source signal as shown in Figure 15 and it was tested under incomplete chaotic data which gave a good estimation hence, we can say it deserved to be under consideration.

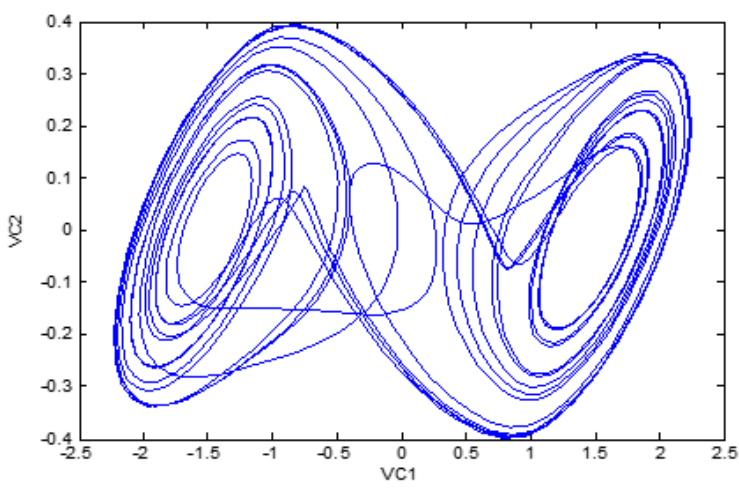

Figure 10. Double scroll circuit

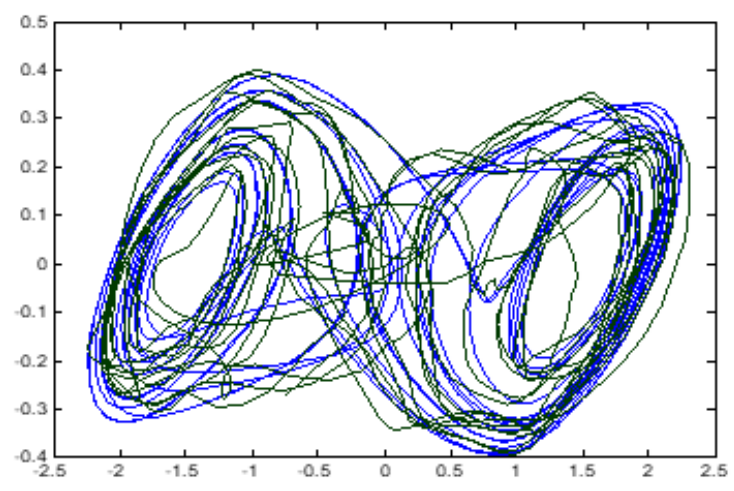

Figure 12. Particles updated in their positions according to the individual nearest objectives

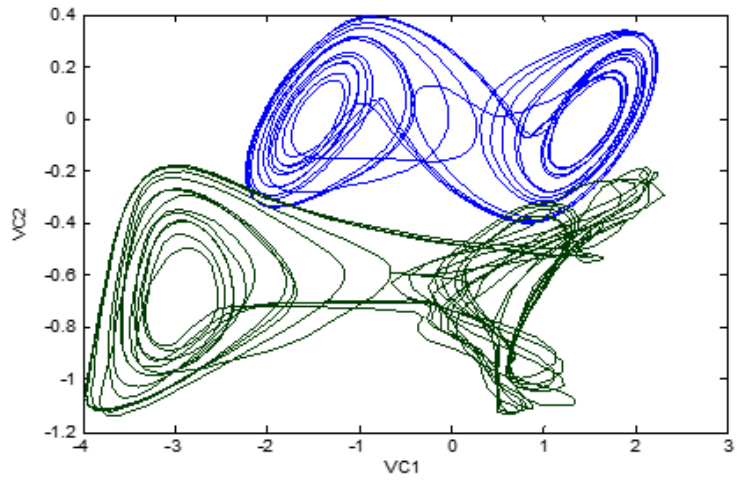

Figure 11. Double scroll circuit

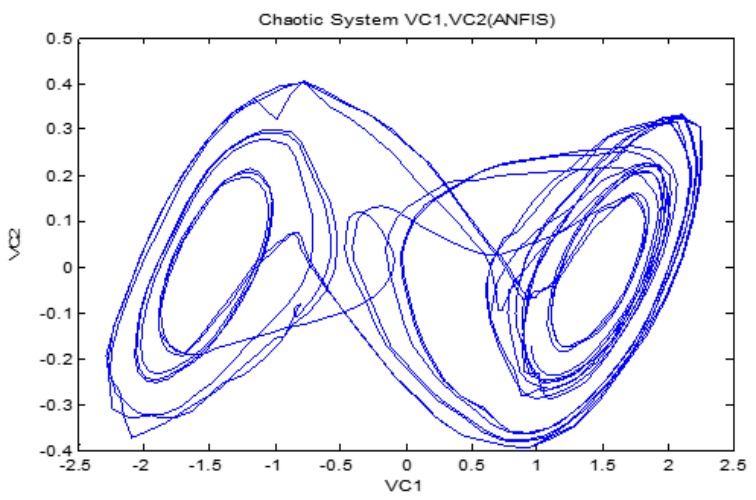

Figure 13. PSO-ANFIS prediction
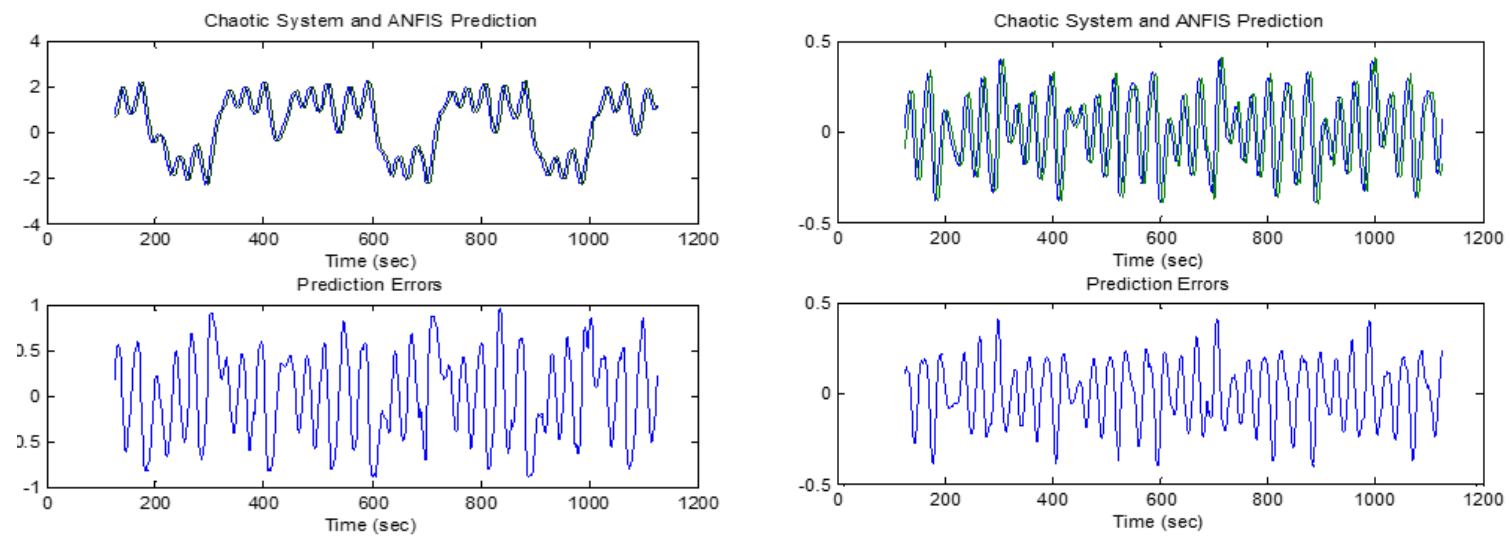

Figure 14. PSO-ANFIS prediction 


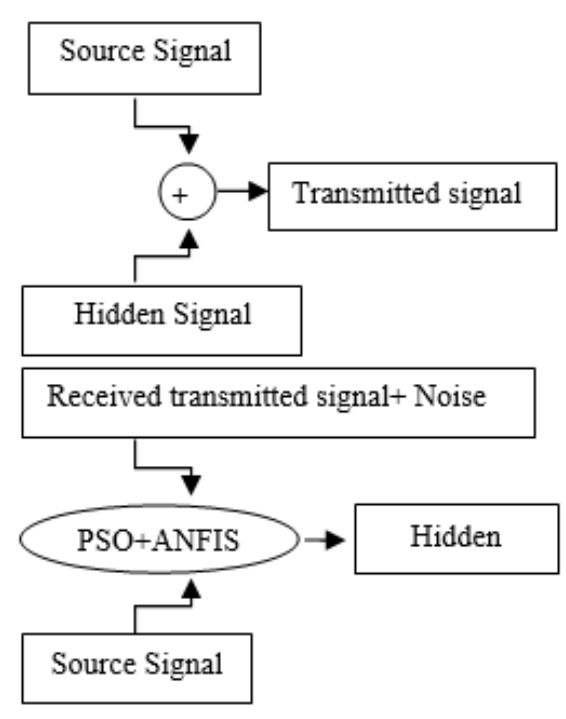

Figure 15. PSO-ANFIS model used for prediction and sperations

\section{CONCLUSION}

However, PSO-ANFIS shows the difficultly to predict the original double scroll attractor when it is distorted, still, it is a good method to recover particles which were absent due to any change and recover the original pattern. This method also can be used to recover the hidden information under the attractor by recognizing the odd particles after they were compared with original attractor data.

\section{REFERENCES}

[1] J. SIDorowich, "Modeling of chaotic time series for prediction, interpolation, and smoothing," IEEE International Conference on Acoustics, Speech, and Signal Processing, vol. 4, pp. 121-124, 1992.

[2] F. Prado, et al., "Forecasting based on an ensemble Autoregressive Moving Average-Adaptive neuro-Fuzzy inference system-Neural network - Genetic Algorithm Framework," Energy, vol. 197, pp. 117-159, 2020.

[3] S. Kar, et al., "Applications of neuro fuzzy systems: A brief review and future outline," Applied Soft Computing, vol. 15 , pp. 243-259, 2014,

[4] M. Malekizadeh, et al., "Short-term load forecast using ensemble neuro-fuzzy model," Energy, vol. 196, pp. 117-127, 2020.

[5] S. Ganjefar, "Optimization of quantum-inspired neural network using memetic algorithm for function approximation and chaotic time series prediction," Neurocomputing, vol. 291, pp. 175-186, 2018.

[6] H. Rad, et al., "Prediction of rock mass rating system based on continuous functions using Chaos-ANFIS model," International Journal of Rock Mechanics and Mining Sciences, vol. 73, pp. 1-9, 2015.

[7] M. Abdollahzade, et al., "A new hybrid enhanced local linear neuro-fuzzy model based on the optimized singular spectrum analysis and its application for nonlinear and chaotic time series forecasting," Information Sciences, vol. 295, pp. 107-125, 2015.

[8] Y.Bodyanskiy, et al., "Hybrid adaptive wavelet-neuro-fuzzy system for chaotic time series identification," Information Sciences, vol. 220, pp. 170-179, 2013.

[9] A. Pano-Azucena, et al., "Prediction of chaotic time series by using ANNs, ANFIS and SVMs," 7th International Conference on Modern Circuits and Systems Technologies, pp. 1-4, 2018.

[10] M. Nhabangue,et al., "Chaotic time series prediction with functional link extreme learning ANFIS (FL-ELANFIS)," International Conference on Power, Instrumentation, Control and Computing, pp. 1-6, 2018.

[11] A. Davies, W. Schwarz, "Nonlinear Dynamics of Electronic Systems," Proceedings Of The Workshop Ndes World Scientific, 1993.

[12] W. Marszalek, el al., "2D Bifurcations and Chaos in Nonlinear Circuits: a Parallel Computational Approach," $15^{\text {th }}$ International Conference on Synthesis, Modeling, Analysis and Simulation Methods and Applications to Circuit Design (SMACD), pp. 1-300, 2018.

[13] B. Samardzic, et al., "Analysis of spatial chaos appearance in cascade connected nonlinear electrical circuits," Chaos, Solitons \& Fractals, vol. 95, pp. 14-20, 2017.

[14] C. Wang, et al, "Capturing and shunting energy in chaotic Chua circuit," Chaos, Solitons \& Fractals, vol. 134, 2020.

[15] G. Leutcho, et al., "Dynamical analysis of a novel autonomous 4-D hyperjerk circuit with hyperbolic sine nonlinearity: Chaos, antimonotonicity and a plethora of coexisting attractors," Chaos, Solitons \& Fractals, vol. 107, pp. 67-87, 2018. 
[16] S. Chung, et al, "Regular sensitivity computation avoiding chaotic effects in particle-in-cell plasma methods," Journal of Computational Physics, vol. 400, 2020.

[17] V. Vaithianathan and J. Veijun, "Coexistence of four different attractors in a fundamental power system model," IEEE Transactions on Circuits and Systems I, vol. 46, pp. 405-409, 1999.

[18] J. Kengne, "Coexistence of chaos with hyperchaos, period-3 doubling bifurcation, and transient chaos in the hyperchaotic oscillator with gyrators," International Journal of Bifurcation and Chaos in Applied Sciences and Engineering, vol. 25, 2015.

[19] D. Karunasingha, et al., "Enhancement of chaotic hydrological time series prediction with real-time noise reduction using Extended Kalman Filter," Journal of Hydrology, vol. 565, pp. 737-746, 2018.

[20] M. AkliKacimi, et al., "New mixed-coding PSO algorithm for a self-adaptive and automatic learning of Mamdani fuzzy rules," Engineering Applications of Artificial Intelligence, vol. 89, 2020.

[21] P. A. Adedeji, et al., "Wind turbine power output very short-term forecast: A comparative study of data clustering techniques in a PSO-ANFIS model," Journal of Cleaner Production, vol. 254, 2020.

[22] M. Rezakazemi, et al., "H2-selective mixed matrix membranes modeling using ANFIS, PSO-ANFIS, GA-ANFIS," International Journal of Hydrogen Energy, vol. 42, no. 22, pp. 15211-15225, 2017.

[23] P. A. Adedeji, et al, "Wind turbine power output very short-term forecast: A comparative study of data clustering techniques in a PSO-ANFIS model," Journal of Cleaner Production, vol. 254, 2020.

[24] M. Ali, Muhlasin, et al., "Combined ANFIS method with FA, PSO, and ICA as Steering Control Optimization on Electric Car," Electrical Power, Electronics, Communications, Controls, and Informatics Seminar (EECCIS), pp. 299-304, 2018.

[25] Y. K. Semero, et al., "PV power forecasting using an integrated GA-PSO-ANFIS approach and Gaussian process regression based feature selection strategy," CSEE Journal of Power and Energy Systems, vol. 4, no. 2, pp. 210-218, 2018. 